\title{
LÍMITE Y RELACIÓN: PENSAR EL CONTACTO DESDE LA FILOSOFÍA DE GILBERT SIMONDON ${ }^{1}$
}

\author{
Marie Bardet \\ IDAES-UNSAM / Paris 8 \\ bardetmarie@yahoo.fr
}

\begin{abstract}
RESUMEN / ABSTRACT
El presente texto propone un recorrido por la obra de Gilbert Simondon a partir de una pregunta acerca de la piel y el contacto. Su conceptualización del límite y de la relación en La individuación a la luz de las nociones de forma y de información permite trazar líneas conceptuales para pensar el contacto en un diálogo con prácticas corporales y artísticas del campo de las técnicas somáticas y de la danza. La lectura de Simondon en diálogo con experiencias sensibles permite entender algunos de los conceptos centrales de su obra y conceptualizaciones originales que se elaboran en el cruce.

Palabras Clave: Simondon, relacionalismo, contacto, danza, somáticas.

\section{LIMIT AND RELATIONSHIP: THINK THE CONTACT FROM THE PHILOSOPHY OF GILBERT SIMONDON}

This text proposes to read the work of Gilbert Simondon from a question for skin and contact. Its conceptualization of the limit and the relationship in L'Individuation à la lumière des notions de forme et d'information allows conceptual lines to be drawn to think about the skin and the contact, and a dialogue to be establish between his philosophy and corporal and artistic practices from somatics and dance fields. To read Simondon's philosophy in dialogue with sensitive experiences allows to understand some of the central concepts of his philosophical work and original conceptualizations that are elaborated in the crossing.
\end{abstract}

KEYWORDS: Simondon, relationalism, contact, dance, somatics.

El presente texto fue escrito a partir de la conferencia "Límites de una relación. O leer a Simondon desde otros lados" pronunciada en el Ier Coloquio Internacional Gilbert Simondon en Argentina, Biblioteca Nacional de Buenos Aires, el 25 de abril 2013. 


\section{Introducción}

$\mathbb{R A}$ La experimentación del contacto con el piso, con otrx o con algún elemento es clave en numerosas prácticas corporales. Así en la técnica Feldenkrais ${ }^{2}$ la atención a los apoyos en el piso es central en una clase, o en las diferentes técnicas de improvisación que, a partir del Contact Improvisation, centran muchas de sus experimentaciones en el contacto y la relación con la gravedad ${ }^{3}$. Técnicas somáticas y técnicas de improvisación ocupan un lugar cada vez más importante en la formación de los bailarines y de las bailarinas, y acuñan fuertemente la producción artística en el escenario de la danza contemporánea o de la performance, que precisamente centran su composición y su propuesta en el contacto. Varios de estos trabajos basados en el contacto consideran la piel como un lugar de intercambio a la vez que un límite. Sus exploraciones se encuentra a menudo con estas "paradojas del pensamiento": por ejemplo, la piel es el límite entre "mi cuerpo" y el piso, y es al mismo tiempo el lugar de intercambio de las fuerzas de gravedad con el piso, dando lugar a una sensación de cierto espesor en ese límite. La piel, Ashley Montagu (2004) nos lo recuerda, es un límite entre yo y el aire que me rodea, al mismo tiempo que es la membrana de intercambio del oxígeno que comparte y hace circular un mismo oxígeno y dióxido de carbono entre "dentro" y "afuera". La piel es producida tanto como produce el contacto; la piel define límite y permeabilidad al mismo tiempo; al mismo tiempo borde y desborde. La piel es un órgano, órgano descentrado por excelencia, órgano desparramado, sin centro ni corazón. Es un órgano membrana, órgano que hace de medio, membrana inter-medio entre adentro y afuera.

En la experiencia sensible del contacto, se expanden las sensaciones y las imágenes de una piel como límite e intercambio de roces, empujes, deslices, en fin, de contactos e intercambios. La piel entra en contacto y se modifica con el contacto, cambia de calor, textura, direcciones... Delimita el adentro del afuera, tanto como es producida por toda esa serie de intercambios entre afueras y adentros. Este carácter productor y producido al mismo tiempo de la piel a través de la relación de contacto es característico de una relación en el sentido exacto de la palabra. Pensar la piel en el contacto como el lugar de una relación es entender cómo un límite puede ser menos delimitante que relacional. Esta problemática es la que anuda una de las propuestas conceptuales fuertes de Simondon cuando emprende su gran proyecto filosófico que consiste en pensar los procesos de individuación (al nivel físico, biológico, síquico y social) sin remitir a priori a los individuos como ya formados, sino a procesos energéticos y materiales de individuación.

2 Una de las llamadas "Técnicas somáticas" (Hanna 1991). Remito a mi experiencia como alumna de esta técnica, y como investigadora de un trabajo que, junto con el grupo de investigación Soma y Po del Laboratorio del Departamento de Danza de la Universidad Paris 8. Ver: Isabelle Ginot (ed.) Penser les Somatiques avec Feldenkrais, ed. Entretemps, 2015.

3 Cf. Marie Bardet, Pensar con Mover. Un Encuentro entre Danza y Filosofia. 
Pensar conjuntamente el concepto de relación y el contacto de la piel, e interrogar los conceptos y los usos actuales de esas prácticas son las dos líneas que guiarán este texto, siguiendo dos ejes problemáticos constituidos por dos palabras que hacen nudo en una lectura entre el original en francés y su traducción al castellano: la relación radical y el límite a la superficie.

\section{Relación radical}

El concepto de un límite menos limitante que relacional funda la perspectiva filosófica de Simondon cuando plantea la idea de relación en el sentido radical de la palabra. Este sentido "radical" de la palabra relación es el que elabora Simondon en $L a$ individuación a la luz de las nociones de forma y de información (Simondon 2015). Va tejiendo, a lo largo del texto, un concepto complejo y multifacético de relación, del cual podemos dar aquí un punto de partida para su comprensión, que proviene de la lectura de la obra en su original en francés y en su versión traducida al castellano, pero que supera la simple anécdota lingüística. En efecto, Simondon establece una diferencia explícita y constante entre rapport y relation, que el traductor al castellano Pablo Ires decide traducir respectivamente por "vínculo" y "relación" para mantener la distinción. La complejidad reside en que tanto rapport como relation se pueden traducir por "relación" en castellano y que las significaciones de cada uno de los términos se solapan ampliamente. Para Simondon, la diferencia es sin embargo radical: el término relation designa lo que no es un rapport meramente "formal" sino que implica una relación propiamente dicha, es decir, recíproca, que transforma el modo de ser de lo que se relaciona, y no relaciona cosas entre sí sino relaciones: la relación [relation] es relación de relaciones. La posibilidad de leer estos textos en dos idiomas permite insistir aquí: la radicalidad de la relación se tiene que escuchar aun cuando relation y rapport se pueden plegar en un solo y mismo término: relación.

Cabe destacar que la radicalidad de esta relación diferenciándose del simple rapport es en La individuación a la vez ontológica y epistemológica. Desde luego, es habitual que el plano epistemológico y el plano ontológico se crucen en Simondon. Así se puede entender el rol que juegan el ladrillo o el cristal en la obra de Simondon: no funcionan como "ejemplos" de una concepción, ni como casos prácticos inspirando una teoría, sino que son los verdaderos operadores conceptuales que permiten a Simondon tejer su filosofía. La individuación está atravesada por una "operación cristal"4 ontológica y epistemológicamente: el cristal viene a formular una ontología (de la individuación), pero también una epistemología. En efecto, el concepto de individuación se va construyendo a lo largo de las extensas, detalladas y repetidas

$4 \quad$ Es en este sentido que podemos hablar de una "operación Cristal" recorriendo todo el libro de La individuación. Ver Marie Bardet, presentación oral del libro de Gilbert Simondon $L a$ individuación a la luz de las nociones de forma y de información, II Edición, ed. Cactus, Buenos Aires. UBA Fac. de Ciencias Sociales - II Coloquio Simondon, 24 de abril 2015. 
descripciones de los modos de operación de los procesos de cristalización. A su vez, toda la construcción de la filosofía de Simondon parece seguir un proceso similar de individuación, y más precisamente, de cristalización, desplegándose no a partir de un centro sino por los bordes, cada borde de un capitulo reaparece como punto de partida en capítulos siguientes, como si cada despliegue temático mantuviera un reservorio de energía ("preindividual" en el sentido en que nunca se despliega del todo a lo largo de un plan de exposición exhaustivo y mucho menos linear). En un apéndice titulado "Forma, Información y Potenciales", incluido en la segunda edición en castellano de La individuación, Simondon llega a reivindicar explícitamente este uso de la operación cristal para el pensamiento:

Habría cambio local progresivo de estatus ontológico del medio: el germen arquetípico primitivo produciría alrededor suyo una primera zona de cristalización; crearía así un modulador un poco más grande, luego ese modulador un poco más grande modularía alrededor suyo, y se agrandaría cada vez más, permaneciendo el límite modulador. Es así que avanza un cristal, cuando se alimenta de un cristal artificial; a partir de un germen cristalino microscópico, se puede producir monocristal de varios decímetros cúbicos. ¿No contendría la actividad del pensamiento, mutatis mutandi, un proceso comparable? Podríamos buscar en particular el fundamento del poder de descubrimiento de la analogía: el hecho de haber resuelto por medio de cierto esquema mental los problemas de un campo limitado de nuestro contenido de pensamiento nos permite pasar transductivamente a otro elemento (...). (Simondon 2015, pp. 507-508)

La relación como concepto central de su filosofía opera entonces tanto en el plano ontológico como en el epistemológico. El concepto de relación [relation], como el de límite (sobre el que volveremos más adelante), tal como aparece en esta cita, sirve para entender cómo son las cosas. Las cosas, en cuanto se individúan, están tomadas en una serie de relaciones en el sentido fuerte de la palabra, es decir, nunca entre cosas propiamente dichas, sino como relación entre relaciones. El mundo está hecho de relaciones de relaciones. Y conocer algo es entrar en relación en el mismo sentido radical de la palabra. Así lo afirma Simondon en la primera parte que versa sobre "La individuación física", uniendo consideraciones sobre los modos de conocimiento y la naturaleza de la relación en sí:

(...) si la relación tiene igual rango de realidad que los términos mismos, como hemos intentado mostrar en los ejemplos precedentes, debido a que la relación no es un accidente en relación con una sustancia, sino una condición constitutiva, energética y estructural, que se prolonga en la existencia de los seres constituidos, entonces las formas a priori de sensibilidad que permiten captar relaciones (...) no crean una irremediable relatividad del conocimiento. Si la relación tiene efectivamente valor de verdad, la relación en el interior del sujeto y el objeto pueden tener valor de realidad. El conocimiento verdadero es una relación, no un simple vínculo [rapport] formal, comparable a la conformidad de dos figuras entre sí. (...) el conocimiento no es una relación entre una sustancia objeto y una sustancia sujeto, sino relación entre dos relaciones una de las cuales está en el dominio del objeto y la otra en el dominio del sujeto. 
El postulado epistemológico de este estudio es que la relación entre dos relaciones es ella misma una relación (Simondon 2015, pp. 89-90).

Simondon despliega la radicalidad de la relación entre consideraciones concretas sobre el modo de individuación (física en este caso) y la epistemología que le permite llevar adelante su estudio que va del ladrillo a la televisión, saltando por los cristales y el espíritu de Horacio, entre muchas otras "operaciones". El pensamiento de Simondon tiene relaciones radicales con cada uno de estos "casos", de estas "operaciones". Este andar del pensamiento, pasando de un campo a otro por los bordes de cada uno más que algún eje central, es el andar, el paso, el ritmo, del pensamiento simondoniano, modulando un campo, luego otro, por relación analógica radicalizada en un modo de conocimiento. Andar pasando de una cosa a otra, ejercer la sensibilidad que permite captar relaciones, conocer relacionándose, pensar desde las relaciones de relaciones y no las relaciones entre cosas, tales son unas de las líneas ontológico-epistemológicas que propone Simondon en su obra; tales son las líneas a seguir para pensar a partir de su pensamiento la cuestión de la piel y del contacto, a partir de los conceptos de relación y límite.

\section{Limite relacional}

[...] como toda modificación de la relación de una partícula con las otras es también una modificación de sus caracteres internos, no existe interioridad sustancial de la partícula. El verdadero individuo físico, aquí también, como en el caso del cristal, no es concéntrico a un límite de interioridad que constituye el dominio sustancial del individuo, sino que está sobre el límite mismo del ser. Este límite es relación, actual o potencial (Simondon 2015, p. 152).

El límite no es la distinción clara entre dos sustancias cuya identificación también clara nos permitiría entender los esquemas y las características de sus relaciones. El límite es el ser mismo, porque es relación actual o potencial, operación misma de la relación, compuesta de actuales y de potenciales. Si La individuación es una invitación a volvernos atentos y atentas a la relación más que a los elementos, o "estar sobre el límite mismo", es en la medida en que sea posible asir la relación sin presuponer una interioridad sustancial de las cosas que se relacionan.

Es lo que permite pensar la piel como un límite y el contacto como una relación, sin presuponer lo que hay dentro y fuera de esta piel; ahondar en la piel como lugar del contacto no sería entonces distinguir los bordes visibles de un adentro centralizado, ni considerar el afuera como punto de vista sobre la piel y su adentro que ésta limita. Para decirlo de otro modo, pensar, con Simondon, la relación y el límite de esta relación, no es pensar los bordes de dos cosas bien definidas que se relacionan, es calzar el límite $\mathrm{y}$ ver hacia donde nos lleva. El lugar de la piel es un inter-medio, que no se define ni como medio interno ni como medio externo. En esta dirección se podrían volver a mirar varios de los aspectos fundamentales de los trabajos en danza que exploran el 
contacto con el piso caminando, rolando, o el contacto con otra persona como en el Contact Improvisation ${ }^{5}$ o en danzas de a dos, como el tango.

Desplazando el foco de un estudio de la piel o de un sujeto tocando un objeto, hacia la relación (y siempre relación entre relaciones: es decir, que considere al "otrx" del con-tacto no como un objeto sino como una relación en sí, o al piso como ya una relación de fuerzas, de consistencias, de lugares y momentos del aquí/ahora, por ejemplo) el contacto despliega mundos de relaciones de relaciones y de límites, que traspasen y hacen estallar la idea de un contacto entre un sujeto interno y un objeto externo.

Toda, o casi toda, clase grupal del método Feldenkrais, llamada clase de "Atención a través del movimiento", empieza con un escaneo de los lugares de apoyos en el piso y una serie de preguntas (sin respuestas) acerca de su cantidad, su cualidad, su forma, su tono, su dinamismo, dolores eventuales, etc. Hace del contacto con el piso, a través de la ropa y de una colchoneta, el campo de emergencia del primer registro de sensaciones de la clase, y hace de la relación con la gravedad una herramienta a la vez organizadora del cuerpo en el espacio y de autopercepción. Este contacto con el piso, muchas veces en posición boca arriba, se constituye como el punto de partida y el lugar a donde volver en momentos clave para sentir si algo cambió en el transcurso de la clase. En este sentido, el método Feldenkrais no apunta a alguna "mejora" del contacto con el piso, sino a hacer de nuestra relación con el piso el efectuador y perceptor de nuestros modos de movernos, apostando a una atención fundamentalmente "diferencial", “dinámica” y “tendencial” (Bardet 2015, p. 202).

En otro estilo, la pequeña danza [small dance] del Contact Improvisation consiste en estar de pie, los ojos cerrados, percibiendo los ya miles de movimientos en curso que acompañan este no hacer nada más que estar en la situación bípeda, las mínimas transferencias de peso de un pie a otro, de adelante hacia atrás. Esta práctica simple se vuelve un hilo rojo que atraviesa muchas de las clases, o de las jams de práctica libre de esta técnica de improvisación desde su nacimiento en 1972.

Basada en el contacto con el piso y con otra persona, el Contact Improvisation apunta a producir movimientos y escuchar al mismo tiempo, a lo largo del desarrollo de dúos que se componen no desde una serie de movimientos pautados o alguna consigna de imagen, de tema o de una parte del cuerpo (que jugarían el papel de "centro" de referencia), sino siguiendo un punto de contacto entre dos bailarines que puede ir derivando hacia arriba, abajo, a cualquier altura o velocidad. Se esboza una atención a lo que se va modificando en el borde, en el límite de la piel:

El resultado de tantos cambios en la orientación espacial y kinestésica en un corto periodo de tiempo me llevó a concebir el espacio como esférico. Esta esfera es

5 Contact Improvisatión es una técnica de danza que emerge de prácticas colectivas en los años 1970 en Estados Unidos. Para una historia de esta corriente, remitimos a dos videos narrando su creación por los propios artistas: Chute y Fall after Newton. Y para una presentación en castellano ver Marina Tampini 2012. 
una imagen acumulada y ensamblada a partir de distintos sentidos, siendo uno de ellos, la vista. Como si mirando rápido en todas las direcciones tuviera una imagen de lo que sería tener una superficie visual a lo largo del cuerpo, en vez de la piel. Pero la piel es la mejor fuente de imagen, porque funciona en todas las direcciones al mismo tiempo. Si pudiéramos apagar la piel, lo apreciaríamos mucho más ${ }^{6}$ (Paxton 1982).

Así habla Steve Paxton, uno de los fundadores del CI, relatando sus experimentos de caída y de saltos en el video "Chute", que narra la historia de los primeros trabajos que dieron lugar al Contact Improvisation. Da cuenta de las perturbaciones en la orientación gravitatoria habitual que provoca el "des-centrarse" en la piel, llegando a una danza que puede tener las patas para arriba y en todas las diagonales. El espacio esférico y lo táctil que toma la posta de la habitual preponderancia de lo visual exigen pensar un límite multidimensional ${ }^{7}$. Exige a su vez pensar un límite relacional en más de un sentido, también porque esa atención conjuga varios sentidos, particularmente lo visual con lo táctil: lo háptico.

Sea pensada a partir de una "a-modalidad perceptiva” según Hubert Godard", o siguiendo la afinidad entre tocar y mirar tal como la describe Paxton, o bien a partir del concepto de lo háptico, es menester concebir cómo mirar y tocar se conjugan y cuánto el contacto deja de ser exclusivamente un tacto de la piel sino que implica todo nuestro equilibrio (Pablo Maurette 2015), las investigaciones sensibles y estéticas del contacto y de la piel dejan en cierta medida de concentrarse sobre la piel y juegan con el descentramiento y la complementariedad entre diferentes sentidos. La perspectiva de una "a-modalidad perceptiva" sostiene que existe un estrato donde las percepciones no están diferenciadas según cada sentido (gusto, oído, tacto, olfato, vista...). Esta idea de "a-modalidad perceptiva" no se refiere a un a priori de la percepción (previo a la diferenciación), ni de un a posteriori (conocido como el fenómeno de sinestesia, cruce entre los diferentes sentidos), cronológicamente hablando, sino a un grado de relación permanente y fundamental entre las modalidades sensoriales que es constitutiva de la percepción misma. De la misma manera, tomar en cuenta las vertientes propioceptivas y kinestésicas del contacto permite ver como se conjuga el tacto con el oído interno y los captores articulares para orientarnos en la gravedad.

$6 \quad$ "The result of so many changing on spatial and kinesthetic orientation in a short time has caused me to understand space as spherical. This sphere is an accumulated image gathered from several senses, vision being one. As if quickly looking in all directions gives me an image of what it would be like to have a visual surface all above my body, instead of skin. But skin is the best source for the image, because it works in all directions at once. If we could turn the skin off, we would appreciate it much more". S. Paxton, "Chute transcript" in Contact Quarterly. Traducción propia.

7 Y no simplemente tridimensional. Sobre este punto en particular, véase: "Lo que siente siendo la manzana" (Bardet y de Ronde 2013).

8 Hubert Godard retoma y amplía el concepto de Daniel Stern (Stern 1989, pp. 70-71,74) donde caracteriza de "transmodal" y "amodal" la percepción del recién nacido. 
En efecto, existe una orientación a través de la gravedad que funciona no solo en términos de localización por referencia a coordenadas fijas (horizontal/vertical, dentro/ fuera) sino como una orientación dinámica a través de los cambios (aceleraciones/ desaceleraciones, acercamientos/alejamientos) que no parten de referencias a puntos de coordenadas, sino que son fundamentalmente relacionales. El tacto en cuanto propiocepción y cinestesia, tacto afín con la mirada, se puede describir como relación entre relaciones; relación entre la relación gravitatoria (ya que la situación de gravedad no es una cosa, sino una relación de fuerzas) y la orientación dinámica de todo mi cuerpo, esqueleto, músculos, fascias, órganos, líquidos, etc., del que también podemos tener una concepción en términos relacionales y no sustanciales. Esto indica rápidamente cómo una perspectiva relacional puede abrir una pista de escape al dualismo sustancial entre alma y cuerpo. Es en este sentido que podemos pensar el despliegue de una atención a través del contacto y la gravedad, como relación de relaciones.

Es precisamente así como lo narra Laurence Louppe en su historiografía original de la danza en Poética de la Danza Contemporánea que concibe una sola danza contemporánea desde fines del siglo 19 (con las obras de Loïe Fuller) que se puede volver a encontrar con más o menos fuerza en cada proyecto artístico a lo largo del siglo 20. En su empresa historiográfica, Louppe dedica parte de su análisis a Laban y su concepción del movimiento a partir de factores y particularmente del "factor peso". Recuerda particularmente el aspecto relacional de la teoría de Laban de la situación gravitatoria del cuerpo humano, "en" movimiento y no fijo, precisamente en cuanto lo describe como "actuando y actuado" [agissant et agi] y saca consecuencias conceptuales singulares sobre la atención que esta concepción sostiene.

Los cuatro factores serán los vectores sensibles de esta elaboración: peso, flujo (es decir, grado de intensidad del tono muscular), espacio y tiempo. Entre estos cuatro factores, el más importante, el peso, ocupa un lugar aparte: es a la vez el elemento ejecutante y ejecutado [l'agent et l'agil del gesto. [...] Pero el peso no solo es desplazado: a su vez desplaza, construye, simboliza a partir de su propia sensación. Los otros factores sirven para definir cualitativamente la sensación de peso y para distribuirla según matices corporales diferentes. De hecho, los cuatro factores no existen en sí mismos: no son aprehendidos bajo el ángulo de su propia sustancia, sino únicamente en la relación que podemos mantener con ellos (Louppe 2011, pp. 91-92).

Es pues en esta perspectiva relacional que se anuda el problema de cómo concebir este límite, esta superficie, esta membrana en contacto, al límite del intercambio de peso.

\section{Atención al límite}

Es allí justamente dónde surge una línea de tensión en la lectura cruzada de Simondon y de estas experiencias en torno al peso y al contacto. Simondon, en su afán de pensar la individuación más que individuos, atribuye precisamente la tendencia que tenemos 
a ver individuos con una interioridad bien definida por oposición a una exterioridad, a la experiencia del cuerpo propio, como individualidad delimitada por la piel, ese envoltorio material que definiría un interior por oposición a un exterior:

Una creencia inmediata en la interioridad del ser en tanto individuo proviene sin dudas de la intuición del propio cuerpo, que parece en la situación de un hombre que reflexiona, separado del mundo por una envoltura material que ofrece una cierta consistencia y delimita un dominio cerrado (Simondon 2015, p. 152).

Es el cuerpo, o mejor dicho la intuición del cuerpo propio, que vendría a inyectar, para Simondon, un sentido de interioridad que transponemos a todas las cosas.

Ahora bien, ¿no se encontraría cuestionada la sensación de lo propio como interioridad y la piel como envoltorio delimitando un adentro separado de un afuera en cualquier mínimo recorrido exploratorio por la piel? Siguiendo el sentido que se venía desplegando de una experiencia de lo táctil como relación, que incluye lo háptico y la a-modalidad perceptiva, se puede pensar como incluidas en el tacto, la propiocepción y la cinestesia que se encuentran formadas e informadas, entre otros, por captores dérmicos, al límite (además de por el oído interno y de captores en los tendones de las articulaciones informando aceleraciones y desaceleraciones).

Es innegable que hay algo táctil en la sensación de trazar algo, en el retortijón, en el latido del corazón, en el dolor de cabeza. En cuanto a la sensación de equilibrio y orientación corporal, la aceleración y desaceleración del cuerpo, se trata de facultades tremendamente complejas que funcionan automáticamente y que, por tanto, es difícil distinguir como sensaciones discretas. Se puede decir entonces que, al menos en un sentido fisiológico, el tacto es el único sentido realmente irreductible. Pero si todas estas formas complementarias de percepción del cuerpo propio se asocian con el tacto antes que con cualquiera de los otros sentidos es porque son, como este, de naturaleza afectiva (Maurette 2015, p. 59).

Entonces, lejos de darnos la envoltura material de un hombre en situación de reflexión, la experiencia de contacto de la piel, más que una conciencia que se repliega sobre sí misma en una re-flexión, se contrae y se dilata, se acelera y desacelera, pone en relación lo de adentro y lo de afuera, y pasa por múltiples pequeñas modificaciones de los contactos con el piso o con otrx, a lo largo y lo espeso de la piel, límite e intercambio a la vez. En efecto, la atención ejercitada a través de la propiocepción como sentido del propio cuerpo en su relación con la gravedad y en la cinestesia como sentido del propio movimiento o más bien, a través del movimiento propio, se basa en la sensación de este límite que es la piel, lejos de remitir solamente a una envoltura. Se despliega una atención a través del movimiento más que una conciencia del cuerpo como objeto inerte; una atención a través de la orientación y sus cambios permanentes. Tal es el tipo de atención que habita y anima gran parte de las prácticas corporales y danzadas desde principios del siglo XX hasta ahora.

Se esboza una concepción del límite, a la altura de una relación propiamente dicha, descentrado, espesado por los intercambios, y produciendo a la vez que producido 
por estos intercambios, por la relación fuera/dentro. En esta perspectiva, pensar desde la alianza entre tacto y gravedad puede ayudar a concebir ese límite sin centro, a lo largo, y producido/produciente, afuerándose/adentrándose más que delimitando un adentro y un afuera a priori. En efecto, el descentramiento de la relación gravitatoria es un aspecto fundamental en la historia de la danza contemporánea. Así lo retoma, lo conceptualiza y lo reivindica un bailarín y coreógrafo como Boris Charmatz quien partió de ese saber de bailarín para fundamentar su propuesta institucional de un Museo de la Danza, transformando el nombre que tiene la institución que dirige "Centro Coreográfico Nacional": "Buscar el "centro"... Para un bailarín, la palabra resuena ante todo físicamente. Hasta hace poco, sistemáticamente se le pedía, a lo largo de su entrenamiento, "encontrar su centro". Pero hoy se admite generalmente que el cuerpo no tiene centro, y que no le hace falta. El cuerpo de la modernidad no necesita centro, pues ese centro ausente, el núcleo que permitía tranquilizarlo, no está, ya no está"9.

Y tal es la tensión que habita el texto de Simondon cuando, un poco más tarde afirma:

En realidad, un análisis psico-biológico bastante profundo revelaría que la relación con el medio exterior, para un ser viviente, no está solamente repartida en su superficie [surface] externa. La sola noción de medio interior, formada por Claude Bernard para las necesidades de la investigación biológica, señala, por la mediación que ella constituye entre el medio exterior y el ser, que la sustancialidad del ser no puede confundirse con su interioridad, aun en el caso del individuo biológico. La concepción de una interioridad física de la partícula elemental manifiesta un biologismo sutil y tenaz [...] Por el contrario, el individuo físico pensado según la relatividad no posee límites propios definidos de una vez por todas por su esencia: no está limitado. [...] El límite, y por consecuencia la relación del individuo no es jamás una frontera; forma parte del ser mismo (Simondon 2015, pp. 152-153)

Así, la experiencia del límite, de la superficie, de la piel, puede dejar de ser (o solamente) la experiencia del borde entre dentro y fuera como dominio cerrado y definitivo del ser, ni de un límite solamente concebido en referencia a un centro. Es todo el concepto de límite que tiene que albergar este esfuerzo, ya que, tal como lo precisa Simondon en muchas partes, haciendo uso de la operación conceptual "cristal": "Importa notar muy especialmente que el carácter de exterioridad o interioridad de las condiciones está modificado por la génesis misma" (Simondon 2015, p. 94).

9 “La recherche du “centre”... Pour un danseur, le mot résonne d'abord physiquement. Il n'y a pas si longtemps, on lui demandait systématiquement, tout au long de son entraînement, de "trouver son centre". Mais aujourd'hui, il est généralement admis que le corps n'a pas de centre, et cela ne lui manque pas. Le corps de la modernité n'a pas besoin de centre, car ce centre absent, le noyau qui permettrait de se rassurer, n'est pas là, n'est plus là". Boris Charmatz (2008). 
Hay que pensar un límite que produce, tanto como es producido, por una relación entre el afuera y el adentro. Invita a que, al momento de tocar y ser tocado por el piso, por un árbol, o por otro u otra, pueda irrumpir más que una simple conciencia reflexiva o hasta fenomenológica: toma consistencia cierto modo de atención no reflexivo, un estar aquí y ahora que desplaza "la situación del hombre que reflexiona". Hace, táctilmente, en el límite, otra cosa que reflexionar a partir de una frontera, o buscar un mejor punto de vista sobre la piel. Tal es una pista conceptual abierta por los conceptos de relación y límite en Simondon, que exigen pensar otros tipos de consistencia, y de superficialidad de este límite en contacto entre dentro y fuera.

\section{¿Limite a la superficie?}

No hay que pensar la consistencia del límite a la superficie o su materialidad específica a partir de la idea de extensión. Si en Descartes la "sustancia" y materialidad se definen conjuntamente por su extensión, en oposición a lo inextenso (res extensa, cuyo carácter extenso funda su oposición con la res cogitans), el gesto simondoniano que anuncia salir de un esquema hylemórfico para pensar la individuación surge al mismo tiempo de una definición de la materia como sustancia extendida. De hecho, leyendo a Simondon, podemos afirmar una solidaridad conceptual entre el dualismo cartesiano y el hilomorfismo y una invitación a alejarse de ambos al mismo tiempo. Y en esta dirección entender la exigencia conceptual a la hora de concebir una surface más allá de saber si es extensa o inextensa. He aquí un indicio en el texto de Simondon para dar un paso más, un indicio que se pliega una vez más en la brecha abierta entre dos palabras en francés que hacen solo una en castellano: cuando Simondon dice, en la cita anterior, que "[e]n realidad, un análisis psico-biológico bastante profundo revelaría que la relación con el medio exterior, para un ser viviente, no está solamente repartida en su superficie [surface] externa" (Simondon 2015, p. 152) usa en francés la palabra surface, que en su diferenciación con el término superficie fuerza a pensar de qué modo esta superficie deja de remitir a un exterior de alguna interioridad. La relación radical que Simondon propone pensar abre a una surface sin superficie. El límite de la relación o la membrana del ser viviente no dejan de ser límite, conservan su carácter de surface, sin tener una superficie en tanto extensión definitoria clara y distintiva. Podríamos decir sobrefaz, pero no está sobre nada.

Si la consistencia viene de un espesor del límite, es en la medida en que deja de ser referida a una profundidad que garantiría el sentido de las cosas, construida en oposición a una superficialidad que carecería de consistencia. De la misma manera, se puede considerar la piel en el contacto en cuanto no consiste en revelar lo profundo, sino en producir/ser producida a través de la dinámica de sus intercambios. Lo que abre la diferencia, plegada en superficie, entre surface y superficie en francés, es el hecho de que esta superficie deja de jugar un papel en la oposición profundidad/superficie. Si surface se aleja de superficie, es en la medida en que se vuelve la superficie de ninguna profundidad, y en que no se define por su oposición a lo profundo. La consistencia del límite de toda relación, tanto la relación-realidad como la relación-conocimiento, 
dejaría de tener el rol de descodificación de lo superficial para conocer la profundidad de una realidad. Habría que pensar una consistencia propia de la relación del orden de un espesor que no remita ni a una profundidad, ni a una superficialidad.

La piel abre a una exploración de superficie [surface] en cuanto tal y no como alguna superficie [superficie] de aparición de los síntomas, pantalla de proyección de las esencias bien escondidas o superficie de inscripción de sentidos profundamente arraigados. La declaración provocadora de Valéry, "lo más profundo es la piel", que Deleuze retoma en su Lógica del Sentido donde la presencia de Simondon es patente, no funciona ni como una mera inversión (lo superficial es profundo y lo profundo es superficial), ni como una abolición (no hay más distinción entre dentro y fuera) sino que da un paso al costado: una afirmación que desplaza volviendo sensible todo lo que las pieles producen y cómo son producidas, por acercamientos-alejamientos, aceleracionesdesaceleraciones, polaridades intensivas que determinan y son determinadas a la vez hacia dentro y hacia fuera.

Veamos una performance de Lygia Clark, artista plástica brasileira, que transitó un cierto camino hacia la performance, o para decirlo en sus términos, que anuncia “desmaterializar" su obra cada vez más, hasta que sus experiencias, que desdibujan las fronteras entre la estética y la clínica, la lleven a "atender" a personas. En una obra que podríamos decir bisagra en este proceso, "Baba Antropofágica" de 1973, propone una larga experiencia de varias horas durante la cual diversas personas desovillan bobinas de hilo que tienen en la boca, recubriendo una persona tirada en el piso con esos hilos de baba y de hilos . Le fabrican poco a poco un ectodermo embrionario, una exodermis informe, que multiplica su membrana descentrada, acercándose a ella por el distanciamiento de un hilo y la intimidad de su baba, a la vez. Una "desmaterialización" que más que una abstracción tiende a un desplazamiento de la materialidad fuera de la oposición entre dentro y fuera, superficie o profundidad, centro o periferia, cercano o lejano. Materialismo y espacialidad de una experiencia de piel, límite, membrana, de un contacto sin tacto de algún borde con otro borde. Resuena una imagen de esta baba antropofágica en la pregunta por cierto espesor de la consistencia de superficie que no remita a ninguna profundidad del sentido.

Esta consistencia que no se agarra ni de una extensión material definida por su oposición punto por punto a una inextensión inmaterial, ni de una centralidad versus sus derivados periféricos, ni de una profundidad opuesta a una superficialidad, exige concebir toda una espacialidad no linear, una temporalidad propia de la membrana, o lo que Deleuze encuentra en Simondon: un mundo de la individuación. Las preguntas por la consistencia de la superficie, o su Lógica del sentido, conducen en este contexto a una serie de reformulaciones de un pensamiento forzado a concebir y a explorar junto con la piel, una surface sin superficie.

[...] las singularidades o potenciales aparecen en la superficie. Todo ocurre en la superficie, en un cristal que no se desarrolla sino por los bordes. Sin duda, no ocurre lo mismo en un organismo; éste no cesa de recogerse en un espacio interior, y de expandirse en el espacio exterior, de asimilar y exteriorizar. Pero no por ello las membranas son menos importantes: ellas llevan los potenciales y 
regeneran las polaridades; ponen en contacto precisamente el espacio interior y el espacio exterior, independientemente de la distancia. El interior y el exterior, lo profundo y lo alto, sólo tienen valor biológico gracias a esta superficie topológica de contacto. Así pues, hay que comprender incluso biológicamente que "lo más profundo es la piel". La piel dispone de una energía potencial vital propiamente superficial. Y, así como los acontecimientos no ocupan la superficie, sino que aparecen en ella, la energía superficial no está localizada en la superficie, sino ligada a su formación y reformación. Gilbert Simondon dice muy bien: "Lo vivo vive en el límite de sí mismo, sobre su límite...La polaridad característica de la vida se encuentra en el nivel de la membrana; es en ese lugar donde la vida existe de manera esencial, como un aspecto de una topología dinámica que mantiene, ella misma, la metaestabilidad por la cual existe... Todo el contenido del espacio interior está topológicamente en contacto con el contenido del espacio exterior sobre los límites de lo vivo; en efecto, no hay distancias en topología; toda la masa de materia viva que está en el espacio interior está activamente presente en el mundo exterior sobre el límite de lo vivo... El hecho de formar parte del medio de la interioridad no significa solamente estar adentro, sino estar del lado interior del límite... El pasado interior y el futuro exterior se enfrentan al nivel de la membrana polarizada..." (Deleuze 1969, p. 79).

Deleuze rastrea una lógica del sentido a flor de piel en las superficies de los inmateriales estoicos, en las superficialidades deslizantes de Alicia en el país de las Maravillas... Con la frase truncada de Paul Valéry "Lo más profundo es la piel" se desplazan al mismo tiempo la superficie y la profundidad central, dejando algo de un espesor del límite cambiante que daría/tomaría sentido por emergencia o despunte. Con su cita agujereada del apartado sobre "topología" de La individuación de Simondon, la superficie no superficial toma consistencia por las polaridades siempre renovadas en el caso de la individuación viviente, y en cierta medida del cristal.

¿Será entonces que esa superficie, al límite, en cuanto lugar/momento de relación de relaciones, es cualitativa por no ser cuantitativa o medible? Este carácter no medible del contorno como dato para pensar el límite surface sin superficie podría ser directamente vinculada con la no medición del alejamiento de un centro en relación con esa superficie. Sin embargo, sigue Deleuze

[...] sería un error pensar que en este contexto la diferencia entre surface y superficie podría pasar por la cuantificación o no, la superficie siendo medible, y la surface teniendo sólo un carácter de límite sin definición métrica. El espesor no es ni profundo ni superficial, ni cuantitativo ni cualitativo. En efecto, esta potencia del límite como surface sin superficie, se encuentra desplegada, precisada, caracterizada, en Simondon a lo largo de las páginas sobre la individuación de formas cristalinas. Se encuentra pues en la individuación del cristal no un caso paradigmático, ni central de lo que sería una demostración, sino más precisamente dice Simondon un "ejemplo liminar (...) [el] más exento de toda inferencia lógica inesencial" (Simondon 1964, p. 106): "un cristal no tiene centro que permita medir el alejamiento de un punto de su contorno exterior en relación con ese centro; su límite no está, en relación con la estructura del cristal, más alejado del centro que los otros puntos; el límite del cristal está virtualmente en cualquier punto, y 
puede aparecer realmente allí a través de un clivaje. Los términos de interioridad y de exterioridad no pueden aplicarse con su sentido habitual a esta realidad que es el cristal" (Deleuze 1969, p. 134).

El cristal es en Simondon, dice Deleuze, algo más que una ilustración, o un caso paradigmático. Es lo que llamábamos una operación, que exige pensar una individuación como relación en el límite, un límite que produce y está producido al mismo tiempo entre interioridad y exterioridad. El cristal como operación real y de pensamiento de un límite como relación, exige pensar la interioridad y exterioridad topológicamente, fuera de toda geometría euclidiana de punto y líneas, centros y distancias.

Todo el contenido del espacio interior está topológicamente en contacto con el contenido del espacio exterior sobre los límites de lo viviente; no existe en efecto, distancia en topología (Simondon 2015, p. 289).

Tal es la historia de límites que se despliega a lo largo de la individuación de Simondon, partiendo de la radicalidad de la relación. El viviente, tanto como el cristal, en el "límite entre la materia inerte y lo viviente" (Simondon 2015, p. 289), fuerzan a pensar en dimensiones topológicas de los límites y no en un espacio euclidiano de las distancias. "Se podría decir que el viviente vive en el límite de sí mismo, sobre su límite; es en relación con este límite que existe, en un organismo simple y unicelular, una dirección hacia dentro y una dirección hacia fuera. En un organismo pluricelular, la existencia del medio interior complica la topología, en el sentido en que hay varias capas de interioridad y de exterioridad (...)." (Simondon 2015, pp. 286-287). Interioridades y exterioridades relativas de las diferentes membranas, al límite, un pliegue de un afuera adentrándose, y viceversa, que lejos de abolir toda diferencia entre interior y exterior, la vuelven relación radical. Es el lugar-momento en que un ser viviente sigue siendo viviente.

[E]sta relación [relation] no es puramente cuantitativa ni puramente cualitativa; es distinta de una relación [rapport] de cualidades o de cantidades; define la interioridad mutua de una estructura y de una energía potencial en el interior de una singularidad. Esta interioridad no es espacial (Simondon 2015, pp. 95-96).

Es necesario pues entender ese desplazamiento de un límite como relación en el sentido fuerte de la palabra y como superficie sin superficie conjuntamente con otro: se desplaza al mismo tiempo lo absolutamente cuantitativo y lo puramente cualitativo.

Queda claro que no podemos resolver esta complejidad diciendo que el contacto como relación en el sentido simondoniano sería una relación con la cualidad de las cosas y no con su cantidad. En efecto, y para volver a la primera distinción, no es que el vínculo sea cuantitativo mientras que la relación sería meramente cualitativa, es más bien toda la repartición entre cualidad y cantidad que se encuentra cuestionada a través de una relación stricto sensu. La relación es un mundo de intensidades que no se dejan resumir por su cualidad o su cantidad, no más que por su centralidad o alejamiento $a$ priori. Por eso necesita de una ontología "topológica". 
Ahora bien, esta topología, que no presume nada de lo lejos o cerca, que introduce espesores y pliegues sin referencias a lo a priori distante o cercano, profundo o superficial, es también lo que permite pensar los pasajes entre campos de pensamiento "vecinos" sin ser ni cercanos ni lejanos. Permitiría pensar algunos aspectos de conceptos clave tales como el de transducción, que aparecía a principio del presente texto, como pasajes en el seno del pensamiento, y es clave en toda la obra de La individuación, o también el concepto de "vecindad" que Deleuze et Guattari (Deleuze y Guattari 2003, p. 208).

Acostada, rolando en el piso, cualquier punto puede pasar al límite, la piel es un órgano, órgano sin centro y tocando a lo largo y a lo espeso de su extensión inextensa, o extendiéndose, se escuchará de otro modo esta descripción del cristal: a través de la piel como surface sin superficie se abren los juegos de recomposición de los contactos, apoyos e intercambios. Una danza donde no solo la punta de los pies toca el suelo, sino que de repente cualquier punto puede entrar en contacto y hacerse suelo, volverse aire. Y si los múltiples ejercicios de rolar en el piso pueden traer algo, es cierto espesor emergiendo a la superficie y cierta redistribución de los lugares que hacen movimiento.

Pliegue y despliegue de una piel que cristaliza, por los bordes, piel de arlequín donde la intensidad no tiene por qué elegir entre extenso e inextenso, entre profundidad y superficie, entre cantidad y cualidad, entre centro y periferia. Exploración de una piel por una lógica compartida de cristalización en tanto contaminación por capilaridad, en tanto con-tacto de un espesor del límite, al límite, en tanto surface sin superficie.

Partir de lo que hay, una situación gravitatoria, varias masas en relación de gravedad y un contacto. Y desde ahí movimientos, imágenes, sensaciones, ritmos y espacialidades hápticas producen y son producidos por contactos: ni un mundo de pura cualidad, ni meras cantidades, sino mundos de concentraciones y dilataciones, aceleraciones y desaceleraciones, que atraviesan al bies y producen sentido escapándose, entre lo profundo y lo superficial, lo cuantitativo y lo cualitativo.

Por debajo de lo continuo y de lo discontinuo existe lo cuántico y el complementario metaestable ${ }^{10}$ (más que unidad), que es el verdadero preindividual. La necesidad de corregir y de acoplar los conceptos básicos en física traduce quizás el hecho de que los conceptos son adecuados solamente a la realidad individuada, y no a la realidad preindividual.

Comprenderíamos entonces el valor paradigmático de los cristales en tanto proceso de individuación: permitiría captar en una escala macroscópica un fenómeno que descansa sobre estados de sistema que pertenecen al dominio microfísico, molecular y no molar; captaría la actividad que está en el límite del cristal en vía de formación (Simondon 2015, p. 13).

10 El concepto de metaestabilidad es otro concepto clave del pensamiento simondoniano, y presupone una posible estabilidad de un sistema que puede incluir zonas inestables. En este sentido asume cierto grado de paradojismo, pues asume que un sistema puede ser inestable y estable al mismo tiempo, en dimensiones distintas. 


\section{Consideraciones finales}

El recorrido efectuado a través la obra de Simondon La individuación, en diálogo con técnicas somáticas y prácticas de danza y performance, permite entender la originalidad de los conceptos de relación y de límite en Simondon, y considerar en toda su envergadura las concepciones teórico-prácticas de contacto como relación a través de la gravedad, atención en el límite a través del movimiento y las relaciones amodales entre los diferentes sentidos que proponen ciertas técnicas corporales y prácticas de danza contemporánea. Finalmente, la cuestión del espesor y de la consistencia de estas superficies no profundas ni superficiales, ni cuantificables ni meramente calificables, nos permite entender cómo las prácticas de danza estudiadas no separan entre poética y técnica física, anclan radicalmente lo imaginario en la sensación, articulan exploración anatómica, técnica de los potenciales de movimiento, y expresión, imágenes y composición.

Nos queda como pista para seguir la invitación a practicar y pensar conjuntamente el gesto en sus direcciones y coordenadas físicas y sus afecciones, en su modo de organización gravitatoria y su poética, en su hacer y en quien lo mira (Godard 1995), y la historia conjunta del rol del peso y de la "Poética de la danza contemporánea" con Louppe:

La importancia del peso es uno de los grandes descubrimientos de la danza contemporánea: no solo el peso como factor de movimiento, según una visión que seguiría siendo planamente biomecánica, sino el peso como elemento poético primordial (Louppe 2011, p. 93).

Lo poético no como un "plus", un suplemento, el momento semiótico por encima del sustrato orgánico, sino tejiéndose con los pesos y los movimientos, las texturas y las líneas de un cuerpo. En este sentido, jaquea fuertemente toda reducción de la cuestión a un cuerpo tomado como objeto, a una oposición entre fisicalidad y poética:

Por nuestra parte, podríamos hablar de "función lírica" que se origina directamente en la presencia del cuerpo en la mutación de sus texturas, de sus fluidos. La inquietud de ver la corporeidad, sus conocimientos, su poética, sometidos a una visión mecánica o puramente biológica (o al menos delimitados en esos modos de representación cercanos al determinismo) es sin duda lo que llevó a toda una corriente del pensamiento en relación con la danza a buscar salidas para una liberación del ser-cuerpo respecto de toda dependencia causalista. Esta búsqueda ha estado presente constantemente en la modernidad de la danza, sobre todo a través de los grandes nietzscheanos (Laban, Humphrey...) que aspiraban al desencadenamiento de las facultades motrices para arrastrar el cuerpo a una dimensión únicamente simbólica. Contrariamente a lo que pudiera creerse, este esfuerzo de arrancamiento no llevó a una negación de las profundidades corporales orgánicas, sino al contrario: al explorar a fondo los resortes más íntimos de la transferencia de peso fue cuando uno y otro hallaron algún elemento de esa poética escondida (Louppe 2011, p. 78). 
En este camino historiográfico de una danza contemporánea, Laurence Louppe atribuye mucha importancia a los efectos de las técnicas somáticas, y en particular en el momento de la Judson Church, tiempo crucial de ebullición experimental colectiva del que emergerán gran parte de las propuestas de las décadas siguientes en los Estados Unidos. Entre ellas el Contact Improvisation que apareció más arriba en las palabras de Steve Paxton, él mismo miembro activo del colectivo que se reúne en un templo transformado en teatro: la Judson Church.

La misma contradicción (aparente) se plantea en las experiencias preliminares al movimiento de rebelión de la Judson Church, donde la utilización de distintos procedimientos de inhibición (tareas impuestas, accesorios constrictores, etcétera) aislarán a su vez al cuerpo-destino de toda la sobrecarga hereditaria, biológica y sicológica. (...) Nada de toda esta libertad de un cuerpo "indeterminado" habría sido posible sin los saberes corporales puestos en práctica desde principios del siglo xx, sin la observación de los diversos estados tisulares, en los distintos lugares de la musculatura, sin la enseñanza de Feldenkrais recibida por Halprin, entre otros; en resumen, sin la referencia de los innumerables "caminos" posibles por los cuales una conciencia errante explora en sus profundidades los circuitos orgánicos, para mejor extraer de todo ello su promesa de libertad y no de sumisión. El cuerpo será en primer lugar lo que pensemos de él, lo que él mismo piense de él, así como el lugar adonde aceptemos que nos lleve (Louppe 2011, pp. 78-79).

Pensar lo más físico junto con lo más poético, pensar la técnica, aquí corporal, junto con los conceptos, hacer cohabitar la filosofía con el arte, autorizarse a estos pasajes de una cosa a otra, saltar con la atención puesta en los modos de hacer, tales son las invitaciones que se pueden encontrar al leer a Simondon; y escuchar, prestar atención a problemáticas y conceptualizaciones de técnicas somáticas ${ }^{11}$ y prácticas de artistas en danza.

Se abren caminos sutiles para dar consistencia a un cruce casi contradictorio, paradójico, como muchas veces el pensamiento más fructífero... Pensamiento inquieto por ciertas dimensiones conceptuales, afectivas, políticas entre lecturas filosóficas y prácticas corporales, de los pensamientos que estas prácticas producen, y particularmente en cuanto contactos y pieles, relaciones y límites abren redistribuciones de numerosas oposiciones binarias: frontera/intercambio; extenso/inextenso; profundidad/superficie; cualidad/cantidad.

11 Para una lectura cruzada de otra técnica somática que es el Body Mind Centering, con la filosofía de Gilbert Simondon, recomendamos el trabajo de Carla Bottiglieri (2010, pp. 246-261). 


\section{Referencias bibliográficas}

Bardet, Marie (2012), Pensar con Mover. Un Encuentro entre Danza y Filosofía. Buenos Aires: Cactus

y Christian de Ronde (2014), “Qué se siente siendo una manzana?”, en E. García y A. Fortunato, eds., Actas de las I Jornadas Internacionales Filosofias del Cuerpo, Cuerpos de la Filosofía. Buenos Aires: UBA; pp. 2633.

(2015), “A Atenção através do Movimento: o método Feldenkrais como disparador de um pensamento sobre a atenção", Revista Brasileira de Estudos da Presenca 5: 191-205.

Bottiglieri, Carla (2010), "D'un sujet qui prend corps. L'expérience somatique entre modes de subjectivation et processus d'individuation", en AAVV, De l'une à l'autre. Composer, apprendre et partager en mouvements, Bruxelles: Contredanse. Ver: http://www.danse.univ-paris8.fr/chercheur_bibliographie. php?cc_id=6ych_id $=34$

Charmatz, Boris (2008), Manifeste pour un musée de la danse. Rennes: CCN. http:// www.borischarmatz.org/sites/borischarmatz.org/files/images/manifeste_ musee_de_la_danse100401.pdf

Deleuze, G. (1969), Logique du Sens, Paris: Editions de Minuit.

Deleuze, G., F. Guattari; P. J. Vázquez y U. Larraceleta (2005). Rizoma: (Introducción). Valencia: Pre-Textos.

Ginot, Isabelle (ed.) (2015), Penser les Somatiques avec Feldenkrais. Paris: Entretemps.

Godard, Hubert (1995), "Le geste et sa perception”, en I. Ginot y M. Michel, La danse au XXème siècle. Paris: Borda.

Hanna, Thomas (1995), "What is somatics?", en DH Johnson, ed., Bone, breath, and gesture: Practices of embodiment. Berkeley, CA: North Atlantic Books; pp. 341-352.

Louppe, Laurence (2011), Poética de la danza contemporánea. Salamanca: Universidad de Salamanca.

Lozano, Rafael Méndez. 27 de julio de 2014. 03 de mayo de $2016<$ http://www. lanacion.com.co/index.php/opinion/item/238611-suicidio-ambiental; >

Maurette, Pablo (2015), El sentido olvidado. Buenos Aires: Mardulce.

Montagu, Ashley (2004), El tacto. La importancia de la piel en las relaciones humanas. Barcelona: Paidós.

Paxton, Steve (1982), "Chute transcript", Contact Quarterly 7: 3-4.

Simondon, Gilbert (2015), La individuación a la luz de las nociones de forma y de información. Buenos Aires: Cactus.

Stern, Daniel (1989), Le monde interpersonnel du nourrisson. Paris: PUF.

Tampini, Marina (2012), Cuerpos e Ideas en danza. Una mirada sobre el Contact Improvisation. Buenos Aires: Cuadernos de Danza - IUNA. 